\title{
THE NATIONAL POLICY FRAMEWORK AND STRATEGY ON PALLIATIVE CARE 2017-2022: WHAT'S IN IT FOR TERMINALLY ILL INMATES IN SOUTH AFRICA?
}

\author{
Chesne Albertus \\ LLB (cum laude) LLM LLD \\ Senior Lecturer, Department of Criminal Justice \\ and Procedure \\ University of the Western Cape
}

SUMMARY

In South Africa many people suffer from serious, incurable health conditions that may render them incapacitated and/or terminally ill. Such persons may inevitably require holistic care such as palliative care. The adoption in 2017 of the National Policy Framework and Strategy on Palliative Care 2017-2022 (NPFSPC) in line with the Health Assembly Resolution 67.19 "Strengthening of Palliative Care as a Component of Comprehensive Care Throughout the Life Course" thus marked a significant milestone for advocates of palliative care, as well as for persons affected by terminal illness. Despite this positive step towards the fulfilment of the right to access healthcare, terminally ill persons who are inmates in South African correctional centres are not sufficiently protected by the NPFSPC. While the policy may be lauded for its detailed provisions aimed at affording appropriate care to free persons, it makes but a few fleeting references to inmates who are terminally ill - a very vulnerable group, given the often appalling conditions in correctional centres, the limited resources generally available to inmates and the stigma attached to them. It is therefore submitted that palliative care should be available to all inmates diagnosed with a terminal illness from the moment they are diagnosed. This article also analyses the stated purpose of the NPFSPC in relation to correctional settings, as well as the impact of disease on correctional centres, and the importance of fostering partnerships.

\section{INTRODUCTION}

During the Department of Health Budget Vote Speech 2017, the Deputy Minister of Health, Dr Phaahla, announced that South Africa had adopted the National Policy Framework and Strategy on Palliative Care 2017-2022 (NPFSPC). ${ }^{1}$ The announcement was encouraging; South Africa had become

Deputy Minister of Health "Budget Vote Speech" (2017) 2. 
one of only a few countries in the world with a policy on palliative care ${ }^{2}$ based on World Health Assembly Resolution 67.19. ${ }^{3}$ A preliminary reading of the NPFSPC, however, unfortunately appears to raise questions as to the State's recognition of its duty to fulfil the right to palliative care of terminally ill inmates. ${ }^{4}$ Consequently, this article highlights the extent to which the NPFSPC deals with palliative care in correctional centres. Furthermore, it analyses the stated purpose of the NPFSPC in relation to correctional settings as well as the impact of disease on correctional centres, and the importance of fostering partnerships.

To contextualise the abovementioned analysis, however, it is necessary first to describe the concept of "palliative care" and to justify briefly why terminally ill inmates are particularly in need of such care. The article then also provides a brief background to the World Health Assembly Resolution 67.19, "Strengthening of Palliative Care as a Component of Comprehensive Care Throughout the Life Course".

\section{PALLIATIVE CARE}

\section{A description}

To some extent, a description of palliative care assists in illuminating the basis for claiming an entitlement to such care. Palliative care includes total care - physical, psychosocial, and spiritual. ${ }^{5}$ "[T] he essence of palliative care is the relief of suffering."6 The World Health Organization (WHO) indicates that palliative care aims to: (a) recognise the importance of life, but regards dying as a normal process; (b) provide relief from pain and other distressing symptoms; (c) integrate the psychological and spiritual aspects of patient care; (d) help patients live as actively as possible until death; (e) assist the family to cope during the patient's illness and their subsequent bereavement when the patient dies; ${ }^{7}(\mathrm{f})$ neither hasten nor postpone death; and $(\mathrm{g})$ use a team approach to address the needs of patients and their families. ${ }^{8}$ The

2 The concept of "palliative care" is described in greater detail later in this article.

3 World Health Assembly "Strengthening of Palliative Care as a Component of Comprehensive Care Throughout the Life Course" (24 May 2014) WHA67.19.

4 According to McQuoid-Mason and Dada A-Z of Medical Law (2011) 414, "terminal illness" as applied to inmates means that the inmate was diagnosed with an illness or condition that will inevitably result in his death. At least two medical practitioners must agree that the inmate is terminally ill. The illness must be incurable. Such a disease or condition must also cause severe physical pain, discomfort and suffering and/or negate a person's prospects of leading a meaningful life. Some patients may be imminently and irreversibly terminally ill. This means that they will die within a relatively brief period of time. Others are regarded as distantly terminally ill because they may have been faced with imminent death at an earlier period but were medically rescued, and are medically sustained for an indefinite period of time. See Fleck "Just Caring: Health Care Rationing, Terminal Illness, and the Medically Least Well Off” 201139 Journal of Law, Medicine and Ethics 156171.

5 Gwyther and Cohen "Legal Aspects of Palliative Care" (2009) http://www.osf.org.za/ Publications/ default.asp?PubCatID=34 (accessed 2016-09-08) 2.

6 Gwyther and Cohen http://www.osf.org.za/Publications/default.asp?PubCatID=34 2.

7 McQuoid-Mason and Dada Medical Law 312.

8 WHO "Non-Communicable Diseases and Their Risks" https://www.who.int/ncds/ management/palliative-care/introduction/en/ (accessed 2016-08-08) 1. 
WHO adds that palliative care is applicable early in the course of the illness, "in conjunction with other therapies that are intended to prolong life, such as chemotherapy or radiotherapy, and includes those investigations needed to better understand and manage distressing clinical complications". ${ }^{9}$ This form of care takes cognisance of the fact that "dying is more than a set of medical problems to be solved. Dying is personal; it is experiential. Caring for people who are dying involves helping them to say and do the things that matter most to them". 10

The NPFSPC assumes all the main elements of the above description and defines "palliative care" as the "holistic multi-disciplinary care of a patient and family affected by a life limiting or life threatening illness and is applicable from the time of diagnosis for all adults and children across the life span and includes bereavement care for the family". ${ }^{11}$ The provision of such care should thus start from the time of diagnosis and should last until the death of a patient. ${ }^{12}$ Though this is the ideal, the application of this principle may prove challenging in the correctional environment where the basic required health assessments upon admission to a correctional centre are sometimes not complied with.

\section{The rationale for palliative care}

Palliative care, as may be gleaned from the above description, is a humane response to persons who may be facing final traumas, heightened grief, and even misgivings about their own existence. In a constitutional democracy like South Africa, palliative care should naturally have been embraced as crucial to upholding the rights to life, dignity and health of the terminally ill. In the absence of such a logical acceptance of palliative care, it becomes necessary to sketch, briefly, the rationale for the claim.

The claim for palliative care cannot be completely detached from the historical attitudes that have inhibited such care as a stage in the right to healthcare. Put plainly, these (often unspoken) attitudes regarding care for the terminally ill have over the years contributed to palliative care being on an unequal footing with the rights to curative and preventative healthcare. This imbalance between the different layers of healthcare may be inferred from the fact that an estimated 50 per cent of all people who die in South Africa "could have benefitted from palliative care"..$^{13}$ By referring to some of these attitudinal barriers, the rationale for granting inmates access to palliative care may become less opaque.

Palliative care is not only about dying, but also about living a quality life (as may be evident from the definition provided above). Despite this understanding of palliative care, it cannot easily be gainsaid that most people choose not to think or talk about such care. The issues that affect

Ibid.

10 Byock "Dying Well in Corrections: Why Should We Care" 20029 Journal of Correctional Health 110.

11 NPFSPC 2017-2022 9.

12 NPFSPC 2017-2022 10

13 Ibid. 
human beings during the period of life called dying - possibly the most vulnerable time in human existence - have thus always been almost unmentionable socially and side-lined in legal discourse. The limited discussions on end-of-life issues arguably exacerbate the vulnerability of those who are affected by terminal illnesses. This is unfortunate and particularly of concern in the South African context where indications are that thousands of people are not enjoying good health and are in fact facing lifethreatening or potentially life-threatening medical diagnosis. At this juncture, it may also be added that health problems are exacerbated in correctional settings. ${ }^{14}$

The lack of, or inadequate engagement with, end-of-life issues may be morally comprehensible as death and the issues related to it are not pleasant or popular subjects of discussion for many individuals. However, cognisance must be taken of the implications of not broaching and dealing with some of these issues. The rights of terminally ill persons and of those affected by their illness are at risk of violation. Such risk may be prevented or mitigated through palliative care approaches.

\section{Why terminally ill inmates?}

When an inmate is diagnosed with a terminal disease while serving time in a South African correctional centre, the issue of release on medical parole is often one of the main considerations. While this is an important consideration, the question of appropriate healthcare should still be prioritised. The following remarks by former inmates may be regarded as an indication that appropriate care is often not afforded to terminally or seriously ill inmates.

"While they waited for the [medical parole] application to be processed they had no access to medication and so they died within the prison premises."15

"The one thing I want, my sister, is at least if one says that they are sick and they really look like they are sick then they must be released. I say this because others they are battling until death. Truthfully I have seen very sick people who had not been released. They had diseases that other people don't have and they end up dying right before us."16

These poignant words of former inmates are perhaps indicative of the maladies affecting inmates. Moreover, they allude to the need for palliative care to be provided to inmates. It should be recognised that access to effective palliative care should be afforded from the moment that a prisoner is diagnosed as terminally ill; whether the inmate is eligible for release should not affect the provision of palliative care. There are thus at least two categories of terminally ill inmate who require care. The first includes those

14 Motala and McQuoid-Mason Medical Law (2013) 1.

15 Albertus The Meaning of the Right to Healthcare of Terminally III Inmates in South Africa (Unpublished LLD 2018) Comment of a former inmate interviewed in the Eastern Cape in October 2016.

16 Albertus The Meaning of the Right to Healthcare of Terminally III Inmates in South Africa Verbatim response of interviewee November 2016, Durban. 
who are not eligible for medical parole, as they do not meet all the requirements of the medical parole provisions. The second category consists of those who have no support outside of the correctional environment and for whom the Department of Correctional Services (DCS) cannot find alternative care in hospice institutions, for example.

A further consideration as to why inmates' right to palliative care requires specific protection is that they do not have the same level of support from others as free persons do. Though state-funded palliative treatment is at present not readily available to everyone who needs it, terminally ill persons who are not imprisoned may, with assistance from their family and support networks, gain access to some state services. They may also depend on their support networks where state assistance is lacking. The plight of inmates is worse. They cannot freely access services available to the public owing to security measures in correctional centres, as well as a lack of transport and staff capacity, and the absence of family or other support networks. Thus, in the absence of state assistance, they are a marginalised group. A policy like the NPFSPC could therefore be crucial to the fulfilment of inmates' right to palliative care, provided that it takes into account the challenges in the provision of healthcare in correctional centres, and offers strategies to address and overcome such roadblocks (discussed in greater detail later). First, however, one of the main motivations for the drafting of the NPFSPC is briefly discussed.

\section{WHA RESOLUTION 67.19: "STRENGTHENING OF PALLIATIVE CARE AS A COMPONENT OF COMPREHENSIVE CARE THROUGHOUT THE LIFE COURSE"}

Though the right to palliative care had not been formally articulated in the early binding international law instruments, the World Health Assembly adopted Resolution 67.19 in 2014. The Resolution is a major milestone as it was the first time that member states had discussed palliative care. ${ }^{17}$ Significantly, South Africa was one of the co-sponsors of the resolution that was adopted by all 194 member states. ${ }^{18}$ Despite unanimous support for the Resolution, the World Health Assembly indicated on 1 August 2017 that globally less than 14 per cent of people who require palliative care receive such care. ${ }^{19}$ Notwithstanding the seemingly stagnant attitude to implementing the Resolution, it is still worth considering how it may assist the plight of terminally ill persons - particularly those who also happen to be inmates.

The Resolution has nine action points that it urges member states to implement. These are as follows:

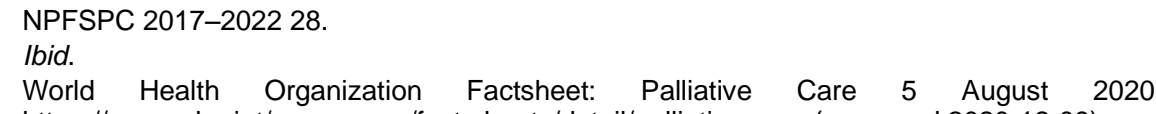


“1) [T]o develop and implement palliative care policies; 2) to ensure adequate domestic funding and allocation of human resources for palliative care initiatives; 3) to provide basic support to families, community volunteers and other individuals acting as caregivers, under the supervision of trained professionals; 4) to aim to include palliative care as an integral component of the ongoing education and training offered to care providers, in accordance with their roles and responsibilities; 5) to assess domestic palliative care needs, including pain management medication requirements, to ensure adequate supply of essential medicines in palliative care; 6) to review and revise national and local legislation and policies for controlled medicines; 7) to update, as appropriate, national essential medicines lists; 8) to foster partnerships between governments and civil society, including patients' organisations, to support the provision of services for patients requiring palliative care; and 9) to implement and monitor palliative care actions included in WHO's global action plan for the prevention and control of noncommunicable diseases 2013-2020."20

The steps envisaged by the Resolution appear to be broad, yet appropriate, allowing member states to form approaches that may accommodate their unique situations domestically. The fact that the Resolution itself does not offer detailed guidance on how to extend palliative care to vulnerable groups, such as inmates, is therefore not a cause for serious criticism. The need to accommodate vulnerable groups ought to be self-evident to states aiming to comply with point 5 above, which calls on member states to assess domestic palliative care needs. It appears, however, that insofar as South Africa is concerned, the particular needs of inmates in correctional centres were not adequately considered. Except for a few fleeting references to inmates and correctional centres, the NPFSPC does not offer any detail on the challenges and possible strategies to address them. Furthermore, it is submitted that South Africa (as discussed later) has also not dealt satisfactorily with point 8 , which calls for the fostering of partnerships.

Point 5 is also an issue of concern in South Africa. The NPFSPC notes that doctors are in relatively short supply in the country $(0.77$ per 1000 population) and that this makes it necessary to shift certain tasks in the provision of palliative care to nurses. ${ }^{21}$ One such shift ought to be that nurses be allowed to prescribe opioids, which are important for pain and symptom management. The NPFSPC acknowledges that the new policy allows nurses to prescribe medication from the Primary HealthCare Standard Treatment Guidelines and Essential Medicines List, but the NPFSPC itself notes that it is unclear if they may also prescribe opioids. Although these are serious concerns, they would be more intense in a prison context where there are additional factors such as drug addiction, security concerns and staffing limitations that may affect the administration of even basic medications. However, these considerations are not mentioned in the NPFSPC.

The adoption of the Resolution and the later introduction of the NPFSPC denote that the State recognises the need for palliative care, and that it has a duty to fulfil this need. While these are positive steps, it must be noted that it took South Africa three years to introduce the NPFSPC after the adoption

NPFSPC 2017-2022 28

21 NPFSPC 2017-2022 25. 
of the Resolution;22 and in its current form, the policy does not sufficiently protect terminally ill inmates' right to palliative care; nor does it introduce reasonable and workable plans to improve their access to such care - as may become clearer below.

\section{THE IMPACT OF DISEASE IN CORRECTIONAL SETTINGS}

It is positive that the NPFSPC acknowledges at the outset that it has become crucial for palliative care to be integrated into healthcare service delivery, given the already heavy burden of disease. ${ }^{23}$ Though it is well known that in correctional settings this burden is even more pronounced than in many other communities, a statement to this effect is absent from the NPFSPC. Due consideration of the full extent of disease and its impact in correctional centres does not emerge from this policy framework. This is further demonstrated by the fact that in expressing the need to expand access to palliative care to all, the NPFSPC provides that "[w]e can only achieve this access if we focus on strengthening services at a primary health care level which includes our clinics as well as care within our communities and in the homes of patients". ${ }^{24}$ The impact of disease on free communities is thus recognised, but no mention is made of its effects in the correctional system. Admittedly, it may be difficult to anticipate how the Department of Health may expand access to palliative care to inmates, but such difficulty does not justify an avoidance of the issue, especially in a national policy framework. If creating equitable access to palliative care for all, including vulnerable inmates, is to be achieved, the extent to which the inmate population is affected by disease should at least have been presented and acknowledged in the NPFSPC, so as to put the issue on the State's agenda.

\section{THE STATED PURPOSE OF THE NPFSPC}

A less obvious, yet serious, concern is that the most significant purpose of the NPFSPC, as stated in the policy framework itself, is to change how palliative care is viewed. ${ }^{25}$ The policy seeks to instil the view that "palliative care includes but does not equate to end of life care". ${ }^{26}$ Changing perceptions on palliative care is not objectionable; it is in fact necessary in the South African context. However, public acknowledgement of the need for palliative care, and education about what such care entails, is not tantamount (nor sufficient) to recognising the fundamental right to such care, and does not automatically indicate the State's duty to provide such care.

The most significant purpose of the policy framework should therefore have been to engender an understanding that the State has a legal duty to

22 The Resolution was adopted in 2014 and South Africa introduced the NPFSPC in 2017 (NPFSPC 2017-2022 2).

23 NPFSPC 2017-2022 2.

24 Ibid.

25 NPFSPC 2017-2022 4.

26 Ibid. 
fulfil towards all bearers of this right. Changing the perception of palliative care as envisaged in the NPFSPC may improve the plight of some terminally ill persons in that a more charitable view may be adopted to creating access to such care for them. However, a more direct and forceful expression is needed to recognise palliative care as the right of all terminally ill persons, as well as the State's duty to fulfil the right. Education and awareness strategies to change perceptions should then be aligned to such expressed right so as to avoid misconceptions about palliative care provision as a state obligation.

\section{FOSTERING PARTNERSHIPS}

It is positive that the NPFSPC affirms that palliative care is currently mainly offered by the non-governmental sector; but the NPFSPC also aims to facilitate the State's responsibility to strengthen health systems in order to extend integrated care, including palliative care for life-threatening or lifelimiting illnesses. ${ }^{27}$ Thus, a reasonably concrete plan for (introducing and) aligning standards of palliative care within correctional centres with those offered in the public domain is a basic expectation. This expectation is further cemented by the NPFSPC's description of various models of palliative care that allow for the provision of palliative care in different settings and according to the needs of a specific community. ${ }^{28}$ Additionally, the NPFSPC provides assessment tools to measure patients' need for palliative care, which would arguably ensure that resources are used appropriately. ${ }^{29}$

Furthermore, the NPFSPC indicates that it aims to make palliative care accessible to everyone, including those who are vulnerable. Terminally ill inmates are included as a vulnerable group. The NPFSPC refers to certain barriers to palliative care in correctional centres. It indicates that palliative care in prisons is challenging because of a "hostile" environment in which access to family is limited and there are not enough carers for terminally ill inmates. ${ }^{30}$ In most correctional centres, nurses are only available during the day, thus making opioid administration impossible at night. The policy also mentions a need for the development of a strategy to establish a multidisciplinary team specialising in palliative care to be consulted on issues regarding medical parole and comprehensive care. ${ }^{31}$

The NPFSPC may be commended, as it offers various models for palliative care in different settings, provides assessment tools to determine patients' need for such care, and identifies some of the problems that impede access to palliative care in correctional centres. Unfortunately, it fails to lay the foundation for partnership with the DCS, although this may be implied in the NPFSPC's mention of some of the challenges to palliative care in correctional centres. However, the policy does not grapple with these

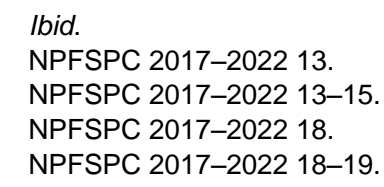


challenges and nor does it provide broad guidelines for addressing such issues.

At best, the NPFSPC sets the following goals:

1. strengthen palliative care services across all levels of the health system, from the tertiary hospital to the patient at home, to provide integrated and equitable care;

2. ensure an adequate number of appropriately qualified healthcare providers to deliver palliative care at all levels of the health service;

3. establish and maintain systems for the monitoring and evaluation of South Africa's palliative care programme;

4. ensure appropriate allocation of financial resources to strengthen and sustain South Africa's palliative care programme; and

5. strengthen governance and leadership to support implementation of the policy. ${ }^{32}$

It is only with regard to goal 5 that the NPFSPC appears to take cognisance of the need to engage the DCS. In pursuit of this goal, an inter-governmental forum will be established to strengthen delivery of palliative care by other sectors, including correctional services. The goal's ultimate target is for all social cluster policies to be supportive of palliative care service delivery by 2022.33

The achievement of the goals of the NPFSPC may greatly assist in finessing, and perhaps even entrenching, the State's duty to provide palliative care to terminally ill persons in the long-term. However, there may be major discrepancies between standards of palliative care in free communities and correctional centres respectively; different policies will have been developed by the different departments with the continuing aim to align such policies, but without any guarantee of achieving it. Although the State generally has a duty to provide palliative care to all terminally ill persons, inmates and free persons may not have access to the same treatment as a result of the parallel creation of separate policies. Resources would therefore have to be spent on aligning policies, as opposed to effectively implementing policies conceived and framed with an ethos of upholding equality between inmates and free persons.

In summary, the NPFSPC is undeniably a positive step towards acknowledging and fulfilling the right to palliative care of all free persons. However, it may widen the gap between inmates and free persons in terms of the State's duty to provide palliative care to both sets of persons. In view of terminally ill inmates' profound vulnerability, the State's duty to fulfil their right to palliative care ought not to be dealt with as a secondary matter that may be deferred. A more prudent approach in drafting the NPFSPC would have been to consider the Guidelines for Palliative Care in Correctional Settings (Guidelines). ${ }^{34}$ The Department of Correctional Services drafted the

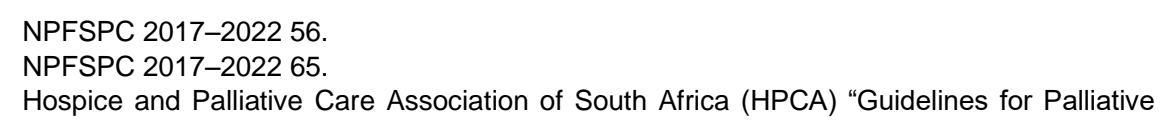


Guidelines with the assistance of the Hospice Palliative Care Association in 2013. Although there is no evidence that the Guidelines have been implemented nationally, their quality provides some indication of the benefit of fostering partnerships. Consequently, some of the guidelines are briefly discussed next.

The unique nature of the correctional environment requires special consideration in the formulation of guidelines. Although conditions may vary in correctional centres, the Guidelines are arguably more enforceable than guidelines drafted for palliative care in the home environment. As there is no specific law or set of regulations on palliative care in correctional centres, the Guidelines provide a list of legislation and policies (22 items in total) that should be complied with in the provision of palliative care. ${ }^{35}$ While it is helpful for correctional authorities to be aware of the laws and policies that they should uphold, they also require explanations as to the exact relevance of these laws and how compliance may be ensured. Despite these considerations, the Guidelines ought to be introduced in correctional settings, as they would caution staff and inmates that the law requires palliative care to be provided and that specific standards must be followed. In the long-term, however, efforts ought to be made to consolidate laws specifically relevant to palliative care in correctional centres, as this may result in greater awareness of the law and perhaps make compliance with such law easier.

The Guidelines provide that an inmate has the right to be involved in any decision relevant to his or her care, treatment and service. ${ }^{36}$ Although this is legally correct and applies to everyone, it is all the more important for it to be emphasised in respect of inmates. They should also be afforded professional support to assist them in making decisions, and be permitted to confer with their next-of-kin if they wish to do so. It is unfortunate that the Guidelines do not say this expressly, and yet this does not render them ineffective, as long as there is a basic understanding that inmates should have a say in how they are cared for.

The Guidelines provide that plans for palliative care ought to be based on a needs assessment of the inmate population, characteristics of the physical institution, medical-care capabilities, and other resources. ${ }^{37}$ These are realistic considerations. If the Guidelines are followed, correctional authorities will have to address the question of how high-quality palliative care may be provided in an oppressive, overcrowded environment, where there is also a lack of reasonable medical resources, as these factors do exist in many prisons. Admittedly, it may be very difficult to address the issues mentioned here, but these are the exact impediments to palliative care that ought to be addressed by the State.

The Guidelines affirm that inmates may have no familial support owing to

Care in Correctional Settings" https://www.hpca.co.za/category/resources.html (accessed 2017-09-20) 1.

35 HPCA https://www.hpca.co.za/category/resources.html 3.

36 HPCA https://www.hpca.co.za/category/resources.html 4.

37 Ibid. 
their incarceration and consequent separation from their biological family. The Guidelines therefore introduce the concept of a "family of choice", which is defined as "biological family members, significant others from the community and/ or inmates or individuals named as family by the inmate patient". ${ }^{38}$ The family of choice is, as would be the case in a community setting, the focus of care and support. This is excellent as some inmates serve long sentences and do form emotional bonds with their peers. If not for this guideline, the importance of affording support to the family of choice may probably not occur to all correctional health staff.

The Guidelines also include further steps that should be undertaken in providing services: staff and management should be sensitive to the culturally and spiritually diverse needs of the correctional community it serves; palliative care should be offered as part of comprehensive care according to the need of each inmate; care must be fully coordinated to ensure continuity from admission to release/parole of the inmate patient and for the family; and a written care plan must be developed for each inmate patient. Such plan must take into account the special needs of the family and the services provided to the family, at the inmate patient's discretion. An interdisciplinary team must identify and incorporate specialised professionals, and must meet the specific needs of inmate patients and their families as identified in the plan of care. They should provide quality, coordinated care as defined by current professional guidelines that relate to the team member's practice specialties and to the principles of interdisciplinary team practice. ${ }^{39}$

The Guidelines, if properly implemented, would ensure that the individual needs of each inmate diagnosed with a terminal disease would be identified and documented. The interdisciplinary team would furthermore ensure that holistic care is provided to the inmate and that the needs of his family of choice are also attended to.

The Guidelines also provide for spiritual care services. ${ }^{40}$ Such services should be based on an initial and ongoing documented assessment of the spiritual needs of the inmate patient and of his or her family by a chaplain or spiritual worker, using required resources as needed. The provision of these services may be of paramount importance to inmates who may be in extreme physical and emotional pain. This is also true for those who may be facing death in the near future. Spiritual guidance may provide them with comfort.

The Guidelines provide that the caregiver services in a correctional centre may include volunteer inmate caregivers, specially trained in the care of inmate patients who are terminally ill. ${ }^{41}$ This guideline may be beneficial to both terminally ill inmates and the volunteer inmates. The former may be comforted by the fact that someone familiar with their struggles and experiences is caring for them, while the latter may acquire new skills that

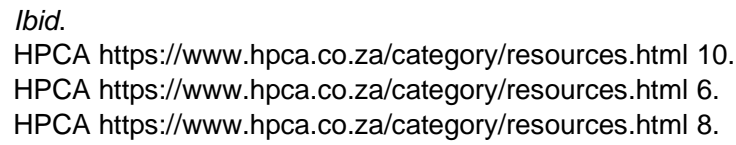


may assist them in finding employment once released.

Further guidelines include: throughout all phases of care, patient needs must be matched with appropriate resources within (and, when necessary, outside) the correctional centre; all patients cared for by the correctional centre should have their holistic needs identified through an established assessment process; and when an opportunity to improve or to correct a problem in the quality of care or services is identified, action must be taken to improve the care or to correct the problem. ${ }^{42}$

The abovementioned guidelines are important. If implemented, they will ensure that the correctional authorities are always aware of the actual needs of terminally ill inmates, as well as the problems they experience. The Guidelines also indicate that the State should make resources available to ensure the care of terminally ill inmates, and where their needs cannot be met within the correctional setting, services that are available to the public must be accessed.

Importantly, the Guidelines suggest that patient accommodation should be adequate to allow for the safety, privacy and comfort of patients. ${ }^{43}$ Furthermore, patient and family educational needs must be assessed and recorded. The correctional centre must design and carry out processes to provide continuity of patient care when a patient is transferred to another organisation; all terminally ill inmates referred for medical placement should have access to the services and support of a palliative care interdisciplinary team.

The Guidelines indicate that terminally ill inmates should not be accommodated in overcrowded cells where they have no privacy, where safety is compromised, and their symptoms may be exacerbated. Creating a comfortable environment conducive to the needs of the terminally ill inmate appears to be vital to ensuring proper care for them. Apparently, an understanding of the needs of the inmate and what palliative care involves is important; hence the educational needs of the family must be assessed and recorded.

Unsurprisingly, the Guidelines provide that the pharmaceutical needs of inmate patients should be met, consistent with all applicable regulations and acceptable standards of practice. Inmate patients should receive coordinated and accurate communication, information, instruction and education about their medication, medication profile and results of medication monitoring. The correctional centre should decide which medicines and pharmaceutical supplies will be made available for prescribing and ordering. This decision is based on patient needs and the types of services provided. ${ }^{44}$

If all the guidelines discussed above were to be followed in all correctional centres, it may lay the foundations for a human-rights-orientated approach to palliative care. A lesson to be learnt from the existence of the Guidelines,

42 HPCA https://www.hpca.co.za/category/resources.html 9.

43 Ibid.

44 HPCA https://www.hpca.co.za/category/resources.html 13. 
which were drafted almost six years ago, is that, though the content is appropriate for correctional centres, a firm undertaking from DCS is needed for actual implementation. There can be little doubt that the Guidelines are not followed in all correctional centres. This may be attributed to conditions in correctional centres, but it cannot be gainsaid that the non-binding nature of this instrument also contributes to the failure to observe it. Arguably, the position of terminally ill inmates would have been bolstered had the Guidelines been given due consideration in the drafting of the NPFSPC. Their claim for palliative care would have been bolstered to some extent.

\section{CONCLUSION}

The time is ripe to recognise that not all terminally ill inmates are released from correctional centres. In the past, some inmates were denied release on medical parole, even in instances where they had been diagnosed with a terminal illness. The problem of terminally ill inmates who require palliative care thus establishes an urgent need for state action. Moreover the State must recognise that it has a legal obligation to provide terminally ill inmates with palliative care. Furthermore, it must be realised that "[i]nitiatives to improve the health of a country's citizens are ineffective if they do not reach those in greatest need". ${ }^{45}$

Though great strides have been made in South Africa to give effect to health needs that can be addressed through curative and preventative measures, a substantial number of citizens require palliative care. Policymakers and legislators must, however, also address barriers to access to the health system as increased expenditure by itself does not necessarily enhance access. ${ }^{46}$ It ought also to be remembered that "[a]t the end of life, we are far more alike than different from one another [and] that [h]ow we care for others may well determine how we are cared for ourselves". ${ }^{47}$ Though infirmity and death are some of the least popular subjects of conversation, it is ever the reality for thousands of people.

The NPFSPC affirmations concerning palliative care as a basic human right do not suffice in engraining such a right in the South African legal system. Admittedly, the NPFSPC is a commendable achievement for advocates of palliative care. As a policy document with a finite duration, it may be the impetus for service delivery until the year 2022. The laudable commitment of the steering committee may make this reasonably achievable. After the five-year period, the steering committee may dissolve, and the officials and focus of the Ministry of Health may also change. The current momentum for creating equitable access to palliative care may consequently decline and support for the right to palliative care, although buttressed by other laws, may have to be summoned and recalled afresh. This may be especially true for terminally ill inmates for whom the right to healthcare (even though constitutionally guaranteed) is often a point of

45 Butler and Plaks "Access to Public Healthcare in South Africa" 2012 South African Actuarial Journal 130.

46 Ibid.

47 Byock 20029 Journal of Correctional Health 110. 
contention. An explicit conferral of a right to palliative care within the framework of an Act of Parliament would guarantee the permanence and further development of palliative care in South Africa. It is therefore asserted in this article that the right of terminally ill inmates to palliative care should be made explicit in national legislation to ensure its permanency, expansion and development beyond the NPFSPC. The introduction of the NPFSPC is a major step towards the formal legal recognition of palliative care as a right for terminally ill persons. Whether or not such recognition will materialise and put palliative care on an equal footing with curative and preventative care for terminally ill free persons will become clearer over time. Currently, however, the NPFSPC signifies that the State accepts that, for a five-year period (the duration of the policy framework), it has a duty to introduce palliative care as appropriate care for terminally ill persons.

In short, overall, great effort and resources have been invested to implement and expand palliative care to public hospitals, local clinics and the homes of terminally ill persons. Although the NPFSPC states a number of times that all terminally ill persons, including inmates, have a right to palliative care, it is unclear how the State will fulfil its duty to them. It also appears that the Department of Health does not envisage a direct role for itself in making palliative care accessible to inmates. 